\title{
Elderly empowerment in ophthalmic surgery: Informed consent and shared decision
}

\section{Nuno Babo"; Júlio Teixeira1; Camile Lanzaro1; Carina Gomes²; Maria João Campos; Maria Lurdes Bela \\ 1 - Serviço de Anestesiologia do Centro Hospitalar e Universitário de Coimbra - CHUC, Coimbra, Portugal \\ 2 - Serviço de Oftalmologia do Centro Hospitalar e Universitário de Coimbra - CHUC, Coimbra, Portugal}

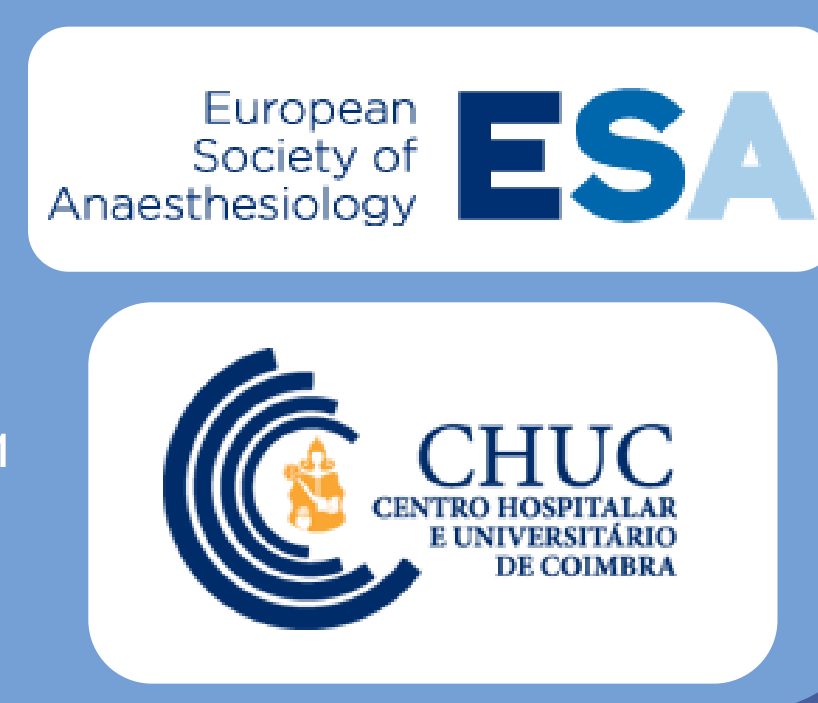

\section{INTRODUCTION}

Empowerment is seen as a contextualized practice in the process of autonomy and self-determination aimed at the patient's involvement and decision-making in the health process ${ }^{1}$

Factors like old age, analfabetism and low cultural knowledge may be obstacles towards an informed decision, and the respect of individual values and patient independency.

\section{OBJECTIVES}

Analyse elderly empowerment in their role of decision-making;

Assess if informed consent applied was participative.

\section{METODOLOGY}

Prospective, observational study. July - November 2017. Ophthalmoloic surgical patients with $\geq 65$ years. Carried out through telephonic interview.

\section{Work instruments:}

\section{Six Item Cognitive Impairment Test (CIT)}

Composed by 6 short questions, non-cultural. Year, month, hour memorize adress with 5 items, regressive countdown of months and numbers from 20 to 0 . Results are scored from 0 to 28 , with a higher score being worse. If $>10$, there's suggestion of cognitive deficit, reason why all of those cases were excuded from the study.

\section{Geriatric Depression Scale (GDS 15)}

15 items of YES/NO answer - evaluates possible depression. Range: 0-4 normal; 5-10 suggestive of depression; 10-15 almost always indicative of depression.

\section{Degner Scale (DS)}

4 questions about ideal patient participation going from the "paternalistic" model to the dynamic one

\section{Questionnaire about decision making involvement}

5 questions with the options, "always", "sometimes" and "never" about the preference in their participation on treatment decision and about the incentive of their doctors and family for that. Also if this participation increases, or not, the probability of success.

Demographic variables: age, sex, scholarship, family and domestic profile and residence

Clinical profile: Chronic disease and depression.

Statistical analysis with $S P S S \circledast$, significant value with $p<0,05$.

Excluded: Urgent surgical procedures, age $<65$ years, refused to answer, CIT>10.

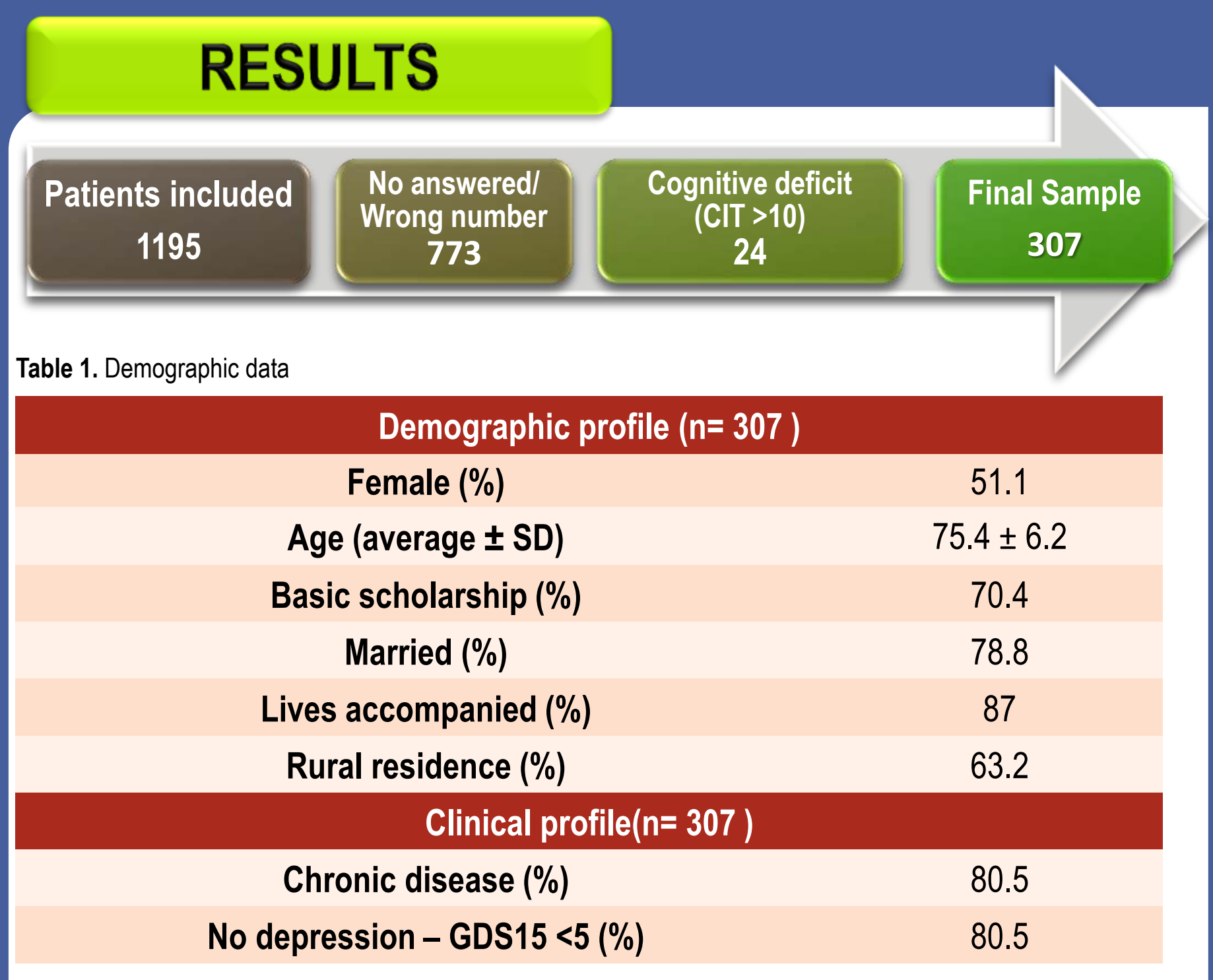

Table 2. Answers from Decision making involvement questionnaire

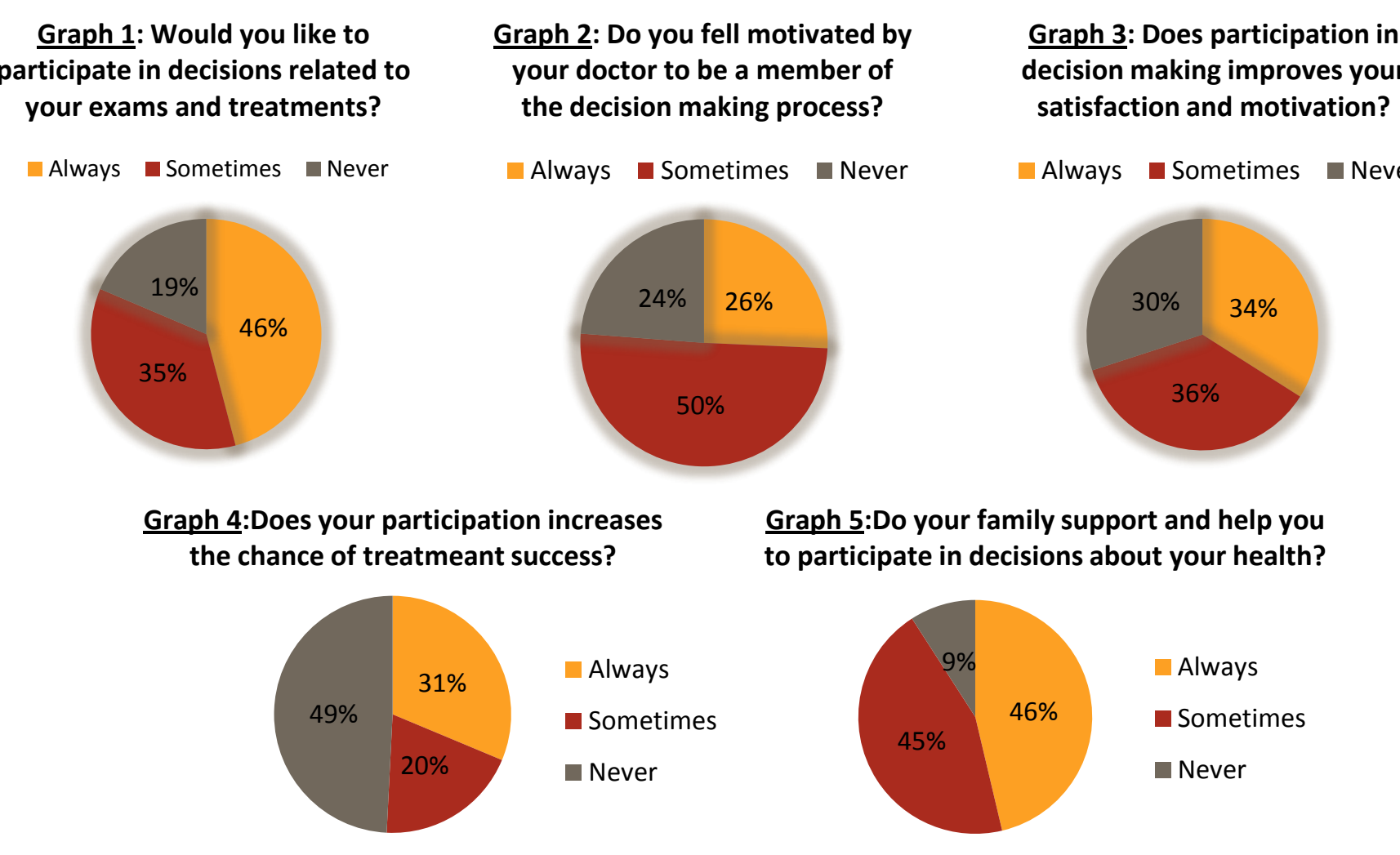

(DS) $\rightarrow 53,1 \%$ rather leave all the decisions related to their treatment to doctors.

Depressed ones (GDS15) answered often they would never like to participate in treatment decision $(p<0,001)$

But, ideally, their aswers show a tendency that they would like the doctors to decide, after their opinion is listened.

Patients with low scholarship $(p<0,001)$, widowers $(p=0,006)$ and rural residence $(p<0,001)$ prefere to leave all the decisions to doctors.

No statistical differences between decision making, gender and chronic disease.

\section{CONCLUSIONS}

High incidence of chronic disease but low number of elderly with cognitive deficit or depression.

Most would like to participate in their's treatment decision because improves their satisfaction, motivation to comply and success of treatment.

Contrary to expected, most rather leaves all the decisions to health care professionals. 